\title{
Síntomas psicopatológicos en adolescentes españoles: relación con los estilos parentales percibidos y la autoestima
}

\author{
Ana I. Rosa-Alcázar*, José L. Parada-Navas y Ángel Rosa-Alcázar \\ Universidad Murcia (España)
}

\begin{abstract}
Resumen: El objetivo de esta investigación fue analizar la sintomatología psicopatológica evaluada con el SCL-90-R en una muestra comunitaria de 935 adolescentes españoles y su interacción con el estilo parental percibido y la autoestima. La muestra estuvo compuesta por 489 chicos y 446 chicas de edades comprendidas entre los 12 y 18 años $(M=14.84, D T=1.83)$ que cursaban estudios de Educación Secundaria y Bachillerato. Catorce centros de la Región de Murcia y Castilla-La Mancha aceptaron participar en el estudio. De éstos, fueron seleccionados al azar siete teniendo en cuenta la titularidad de los centros (pública y concertada). Los resultados indicaron la existencia de diferencias debidas a la edad en todas las variables medidas (síntomas psicopatológicos, autoestima y percepción de los estilos educativos parentales). Las mujeres presentaron menos niveles de sintomatología y mejor percepción de los estilos educativos. Los resultados de los análisis correlacionales confirmaron relaciones significativas y negativas entre autoestima y síntomas psicopatológicos y entre algunas dimensiones positivas de los estilos educativos. Los predictores más relevantes y de mayor peso en la salud psicológica fueron: autoestima, edad, sexo, revelación y afecto del padre y control psicológico de la madre.

Palabras clave: Estilos parentales percibidos; SCL-90-R; autoestima; adolescentes.
\end{abstract}

\section{Introducción}

La preocupación por la salud/enfermedad en niños y adolescentes ha suscitado el interés en los clínicos e investigadores y ha llevado a la realización de estudios epidemiológicos (Schwab-Stone y Briggs-Growan, 1998), etiológicos (Krueger y Piasecki, 2002), de evaluación (Lemos, 2003), tratamiento (Boix, Rosa-Alcázar, Olivares-Olivares, 2012), etc. Desde el modelo de la Psicopatología del Desarrollo (Cichetti y Rogosch, 2002), el análisis de las variables influyentes tanto personales como contextuales en el origen y/o mantenimiento de los problemas sigue generando investigaciones. Dentro de los factores contextuales, la familia es uno de los agentes que, en bastantes ocasiones, puede influir en el aumento, mantenimiento o disminución los síntomas psicopatológicos (Gracia, Lila y Musitu, 2005; Lemos, 2003). La mayoría de los estudios sobre la familia se ha centrado en los padres y, más en concreto, en los estilos educativos de éstos. Se han estudiado los componentes de los estilos educativos y su clasificación (Baumrind, 1968, 1971; Maccoby y Martin, 1983; Pérez y Canovas, 1994; etc.). Estos enfoques tipológicos se han ido complementando con otras investigaciones que consideran a los estilos educativos como un continuo o graduación en una serie de dimensiones. En concreto, en España, Oliva, Parra y Arranz (2008) proponen estilos parentales relacionales caracterizados por el apoyo, el afecto, la

\section{* Dirección para correspondencia [Correspondence address]:}

Ana Isabel Rosa-Alcázar. Departamento de Personalidad, Evaluación y Tratamiento Psicológicos. Facultad de Psicología, Campus Universitario de Espinardo, Universidad de Murcia, Apdo. 4021, 30080 Espinardo, Murcia (España). E-mail: airosa@um.es
Title: Psychopatologic symptoms in Spanish adolescents: relationship with perceived parental styles and self-esteem.

Abstract: The purpose of this research was to study the presence of psychopathologic symptoms in a community sample of 935 Spanish adolescents, and its relationship with perceived parental styles and the selfesteem. Numbers of males and females were 489 and 446, respectively. Age ranged from 12 to 18 years $(M=14.84, S D=1.83)$. Fourteen High School centers in Murcia and Castilla-La Mancha agreed to participate in the current study. Five of them were randomly selected to collected data. The results showed age-related differences in all variables (the psychopathologic symptoms, self-esteem and perceived parental styles). On the other hand, females showed less psychopathologic symptoms and better perception of parental styles than males. Significant negative correlations were found between self-esteem, psychopathology and some positive dimensions of parental style. The main predictors of adolescent's mental health were: selfesteem, age, gender, revelation and affect from father and psychological control of mother.

Key words: Perceived parental styles; SCL-90; self-esteem; adolescents comunicación y la promoción de la autonomía desde una perspectiva más multidimensional.

Las investigaciones centradas en relacionar estilos educativos con diversas psicopatologías, tales como depresión, ansiedad, trastorno de angustia, trastorno obsesivocompulsivo, hostilidad, etc., son variadas. Algunas de ellas han analizado la importancia del control psicológico como variable predictora de los problemas internalizantes, mientras que el control conductual lo era de los problemas externalizantes (Barber, Olsen y Shagle, 1994). Kerr y Stattin (2000) indicaron que la revelación espontánea de los niños se relacionaba con un buen ajuste, mientras que los esfuerzos de control y la vigilancia de los padres estaban vinculados a una mala adaptación. Otros autores se han centrado en la importancia de los problemas de comunicación entre padres e hijos adolescentes, concluyendo que éstos estaban relacionados con la presencia de síntomas depresivos, ansiedad y estrés (Fieldt, Diego y Sanders, 2001; Liu, 2003). Estévez, Musitu y Herrero (2005) indicaron la vinculación entre estos problemas y el ajuste escolar en la salud mental de los adolescentes (síntomas depresivos ansiedad y estrés). Sáez y Rosselló (2001) detectaron que la aceptación de los padres y las críticas familiares se relacionaban con depresión y problemas de conducta en los adolescentes, siendo la aceptación de los padres la de mayor peso predictivo. Ramírez (2002) observó que el afecto negativo correlacionaba con la conducta agresiva, los problemas de atención y del comportamiento mientras que el estilo autoritario lo hacía con depresión y ansiedad, conducta delictiva y problema internalizantes. Hasebe, Nucci y Nucci (2004) relacionaron el excesivo control parental sobre los adolescentes con síntomas internalizantes, destacando que la psicopatología de los mismos 
correlacionaba con un mayor control en el ámbito o dominio privado de los chicos, no tanto en otros campos. Muris, Meesters, Morren y Moorman (2004) encontraron que bajos niveles de calidez emocional y altos niveles de rechazo, control e inconsistencia por parte de los padres correlacionaron con altos niveles de ira y hostilidad entre los adolescentes. Gracia et al. (2005) hallaron relación entre el rechazo parental percibido por el hijo y síntomas de ansiedad, depresión, aislamiento social, autoestima negativa, problemas somáticos, agresividad y conducta disocial. Richard de Minzi (2005) encontró que las familias democráticas promovían afrontamientos adaptativos y protectores ante la depresión y la soledad, mientras que las familias autoritarias generaban inseguridad, afrontamientos desadaptativos y depresión. Heider, Matschinger, Bernet, Alonso y Angermeyer (2006) analizaron la relación entre los estilos parentales y los trastornos de ansiedad y depresivos en seis estados europeos, encontrando una vinculación homogénea en los diferentes países. Los principales resultados fueron los siguientes: el bajo cuidado paternal y maternal obtuvo la asociación más significativa con la presencia de trastornos emocionales, se encontró una correlación positiva entre sobreprotección materna y alteraciones emocionales, no hallándose tal asociación en el caso de la sobreprotección paterna. Oliva (2006) llevó a cabo una revisión de estudios sobre relaciones familiares y desarrollo del adolescente destacando la importancia de la revelación y el control en el ajuste del chico, siendo el control psicológico una variable relacionada negativamente en el desarrollo de la autonomía e identidad, con altos niveles de ansiedad y síntomas depresivos. Posteriormente, Oliva, Parra, Sánchez y López (2007) estudiaron las relaciones entre estilos parentales y ajuste del adolescente (problemas internos y externos), destacando la relación existente entre afecto, promoción de autonomía, revelación y humor y buen ajuste, en concreto menos problemas externos; mientras que el control psicológico estaba asociado al desajuste interno y externo. Además, indicaron que los chicos y chicas que percibían más afecto en sus padres y se comunicaban mejor con ellos presentaban mejores puntuaciones en desarrollo psicosocial, bienestar emocional y ajuste, y que existían diferencias significativas a la hora de percibir a los padres y madres, siendo la percepción de las chicas más alta en algunas de las dimensiones maternas (promoción, control conductual, revelación) y paternas (control conductual y humor). Iglesias y Romero (2009) indicaron una relación significativa entre la baja aceptación e implicación parental y la depresión adolescente y entre el estilo autoritario y las alteraciones externalizantes. Cuervo (2010) presentó una revisión de estudios entre las pautas y los estilos de crianza y el desarrollo socioafectivo durante la infancia y adolescencia concluyendo que la familia puede ser un elemento clave en la prevención de problemas de salud mental en la infancia como depresión, agresividad, baja autoestima y ansiedad, entre otras.

De igual modo, los estudios llevados a cabo sobre la autoestima nos indican correlaciones negativas con síntomas depresivos, encontrando diferencias en cuanto al sexo
(González y Rodríguez, 1993), mientras en otros casos no existen diferencias en esta variable (Bragado, Hernández, Sánchez y Urbano, 2008). Se ha relacionado la autoestima con el consumo de sustancias (Jiménez, Musitu y Murgui, 2008), trastornos alimentarios (Moreno, González y Ortiz 2009), problemas de satisfacción corporal (Taylor, Wilson, Slater y Mohr, 2012), conductas de riesgo (Florenzano et al., 2011; Musitu, Jiménez y Murgui, 2007), síntomas psicopatológicos (Dowd, 2002; Garaigordobil, Durá y Pérez, 2005; Garaigordobil, Pérez y Mozas, 2008; Kim, 2003 etc.). Kim (2003) encontró puntuaciones más altas en sensibilidad interpersonal, depresión, ansiedad y hostilidad pero no halló diferencias en edad y sexo. En el estudio de Garaigordobil et al. (2005) los varones alcanzaron puntuaciones más altas en autoestima, siendo los varones y mujeres con alta autoestima los que menores síntomas psicopatológicos presentaron. Tres años más tarde, Garaigordobil et al. (2008) encontraron diferencias significativas asociadas a la edad en autoconcepto y número de síntomas psicopatológicos, mientras que el sexo correlacionó con los síntomas psicopatológicos alcanzando puntuaciones superiores las mujeres en varios trastornos (somatización, sensibilidad interpersonal, depresión, ansiedad, ansiedad fóbica y cantidad total de síntomas). Los resultados de los análisis correlacionales confirmaron relaciones significativas y negativas entre autoconcepto y autoestima y síntomas psicopatológicos.

Partiendo de las investigaciones previas, el objetivo general de nuestro estudio ha sido analizar la interacción de dichas variables y los síntomas o respuestas psicopatológicas en una muestra comunitaria de adolescentes españoles. Los objetivos específicos planteados fueron los siguientes: (1) Analizar la existencia de diferencias en los Síntomas psicopatológicos evaluados con el SCL-90-R, la Autoestima y los Estilos parentales percibidos debidas a variables sociodemográficas (sexo, edad, tipo de hogar -monoparental, biparental); (2) Evaluar la relación entre los Estilos parentales percibidos, la Autoestima y los Síntomas psicopatológicos; (3) Examinar la capacidad predictiva de las variables mencionadas en la salud psicopatológica de los adolescentes evaluada por ellos mismos. Las hipótesis propuestas han sido: (1) Los Síntomas psicopatológicos, la Autoestima y los Estilos parentales percibidos de los adolescentes presentarán diferencias atendiendo a la edad y el sexo, (2) La Autoestima, los Estilos parentales percibidos y las Respuestas/síntomas psicopatológicos mencionados correlacionarán significativamente, de modo que a mayor Autoestima y mejores Estilos parentales percibidos menores puntuaciones en los Síntomas psicopatológicos. (3) La autoestima, el control psicológico y la revelación percibida por los adolescentes serán variables predictoras de los síntomas psicopatológicos en los adolescentes. 


\section{Método}

\section{Participantes}

La muestra estuvo formada por un total de 935 adolescentes (489 chicos y 446 chicas) con edades comprendidas entre los 12 y los 18 años $(M=14.84, D T=1.83)$ que cursaban estudios de Educación Secundaria y Bachillerato en centros públicos (cuatro) y concertados (tres) de la Región de Murcia y Castilla-La Mancha. El 86.3\% de los chicos/as convivían con ambos padres y el $11.3 \%$ sólo con la madre. En cuanto al número de hermanos, la mayoría tenían dos $(53.7 \%)$ y tres $(20.5 \%)$ hermanos, siendo un $49.6 \%$ de la muestra primogénitos, mientras que un $35.8 \%$ ocupaban el segundo lugar. El $92.6 \%$ de los padres y el $66.8 \%$ de las madres se encontraban trabajando fuera del hogar. El 84.2\% de los padres estaban casados y el $13 \%$ separado o divorciado. El 97\% eran españoles, el 2\% suramericano y el 1\% de Europa central. La Tabla 1 recoge una descripción más exhaustiva de las variables sociodemográficas.

Tabla 1. Descripción de variables sociodemográficas.

\begin{tabular}{|c|c|c|c|}
\hline & & \multicolumn{2}{|c|}{ Frecuencia Porcentaje } \\
\hline Sexo & Mujer & 446 & 47.7 \\
\hline Varón & & 489 & 52.3 \\
\hline $\mathrm{N}^{\mathrm{o}}$ Hermanos & Ninguno & 83 & 8.9 \\
\hline Uno & & 85 & 9.1 \\
\hline Dos & & 502 & 53.7 \\
\hline Tres & & 192 & 20.5 \\
\hline Cuatro o más & & 73 & 7.8 \\
\hline Curso & $1^{\circ} \mathrm{ESO}$ & 171 & 18.3 \\
\hline $2^{\circ} \mathrm{ESO}$ & & 160 & 17.1 \\
\hline $3^{\circ} \mathrm{ESO}$ & & 156 & 16.7 \\
\hline $4^{\circ} \mathrm{ESO}$ & & 155 & 16.6 \\
\hline $1^{\circ}$ Bachillerato & & 147 & 15.7 \\
\hline $2^{\circ}$ Bachillerato & & 146 & 15.6 \\
\hline Estado Civil Padres & Casados & 787 & 84.2 \\
\hline Divorciados/Separados & & 122 & 13.0 \\
\hline Viudos & & 14 & 1.5 \\
\hline Solteros & & 12 & 1.3 \\
\hline Hogar & Biparental & 807 & 86.3 \\
\hline Monoparental madre & & 106 & 11.3 \\
\hline Monoparental padre & & 12 & 1.3 \\
\hline Otros (abuelos, tíos) & & 10 & 1.1 \\
\hline
\end{tabular}

\section{Procedimiento}

Este trabajo se incluye dentro de un proyecto de investigación más amplio sobre la relación entre respuestas psicopatológicas en los adolescentes y variables personales, familiares y contextuales influyentes. El procedimiento utilizado fue el siguiente: (1) Envío de una circular a los centros educativos de Educación Secundaria y Bachillerato de las ciudades de más de 15000 habitantes de la Región de Murcia y Castilla-La Mancha solicitando la participación en esta investigación. (2) Entrevista con los directores de los 14 centros que manifestaron su interés en participar. (3) Selección al azar de siete centros de los 14 anteriores teniendo en cuenta la titularidad pública o concertada. (4). Selección al azar de un aula por curso (en aquellos centros con doble o triple línea). (5) Envío de una circular a los padres solicitando la autorización para que su hijo/a participara en nuestra investigación. (6) Recibida la autorización, se procedió a la evaluación de los alumnos. Éstos cumplimentaron los cuestionarios de forma anónima en una sesión de una hora de duración en presencia de su profesor y un miembro del equipo de trabajo, experto en evaluación clínica. Los adolescentes no recibieron ningún tipo de refuerzo material por su participación. Durante el desarrollo del trabajo se respetaron los principios éticos de la investigación con seres humanos, procurándose las condiciones necesarias para proteger la confidencialidad de los participantes.

\section{Instrumentos de evaluación}

Los participantes cumplimentaron los siguiente cuestionarios: (1) Escala para la evaluación del Estilo parental (Oliva et al., 2007). Consta de 41 ítems en los que el adolescente evalúa de forma independiente 6 dimensiones del estilo materno y paterno. Las dimensiones y su fiabilidad media, calculada mediante la prueba Alpha de Cronbach, fueron las siguientes: Afecto y comunicación $(\alpha=.92)$, Promoción de autonomía $(\alpha=.88)$, Control conductual $(\alpha=.82)$, Control psicológico (dimensión claramente negativa- $\alpha=.86$ ), Revelación $(\alpha=.85)$ y Humor $(\alpha=.88)$. Los índices de fiabilidad en nuestro estudio oscilaron de .80 a .92. (2) Escala de Autoestima (Rosenberg, 1965). Se trata de una escala unidimensional compuesta por 10 ítems que sirve para evaluar la autoestima global de adolescentes. Los resultados de validación de esta escala en universitarios españoles muestran una alta consistencia interna y estabilidad temporal tras cuatro semanas de intervalo (Martín-Albo, Núñez, Navarro y Grijalbo, 2007). El alfa de Cronbach en nuestro estudio fue de .85 . (3) Cuestionario de Sintomas Revisado (SCL-90-R; Derogatis, 2002; adaptación española de González, De las Cuevas, Rodríguez y Rodríguez, 2002). Es un cuestionario autoaplicado compuesto por 90 ítems que permite evaluar nueve dimensiones psicopatológicas (somatización, obsesión-compulsión, sensibilidad interpersonal, depresión, ansiedad, hostilidad, ansiedad fóbica, ideación paranoide, psicoticismo), así como tres escalas generales, que son el Índice Global de Gravedad (GSI), el Total de Síntomas Positivos (PST) y el Índice de Distrés de Síntomas Positivos (PSDI). Los índices alfa de fiabilidad de la adaptación española oscilaban entre .77 y .90 . Los índices de fiabilidad en nuestro estudio variaron de .79 a .85 .

\section{Análisis de datos}

El análisis de datos se llevó a cabo usando el paquete estadístico SPSS (V.20.0.) En primer lugar se realizaron Análisis de Varianza (pruebas post-hoc en caso de resultar significativos), Pruebas $T$ de comparación de medias independien- 
tes y pruebas Chi-Cuadrado de Pearson para comprobar si las puntuaciones en las dimensiones psicopatológicas eran diferentes atendiendo a las variables sociodemográficas. También se calcularon los Tamaños del Efecto -TE, $d$ (diferencia media tipificada, Hedges y Olkin, 1985) entre las mujeres y los varones. Los valores $d_{+}$indicarían mayor puntuación en psicopatología en las mujeres que en los varones. Posteriormente se analizó la relación entre los síntomas psicopatológicos y los Estilos parentales percibidos y la Autoestima mediante los coeficientes de correlación de Pearson. A continuación se efectuaron Análisis de regresión por pasos para examinar la capacidad predictiva de los Estilos parentales y la Autoestima sobre las dimensiones psicopatológicas.

\section{Resultados}

Síntomas psicopatológicos, Autoestima y Estilos parentales percibidos $\mathrm{y}$ variables sociodemográficas

En la Tabla 2 podemos observar la existencia de diferencias debidas al sexo en la mayoría de las respuestas o Síntomas psicopatológicos, excepto en Sensibilidad interpersonal $(p=.353)$, Ansiedad fóbica $(p=.832)$ y Psicoticismo $(\phi=$ $.077)$, siendo más altos los valores medios alcanzados por los varones frente a las mujeres. Igual sucedió con el Índice de síntomas positivos -PST $(p=.232)$. No obstante, teniendo en cuenta los TEs podemos indicar que las diferencias son

Tabla 2. Resultados de comparación de medias en el SCL-90-R atendiendo al sexo.

\begin{tabular}{|c|c|c|c|c|c|c|c|}
\hline & Sexo & $N$ & Media & $D T$ & $T(g l)$ & $p$ & TE \\
\hline \multirow[t]{2}{*}{ Índice Global Gravedad } & Mujer & 446 & 55.09 & 10.51 & \multirow{2}{*}{$-3.92(924,48)$} & \multirow[t]{2}{*}{.000} & \multirow[t]{2}{*}{-.256} \\
\hline & Varón & 483 & 57.99 & 12.00 & & & \\
\hline \multirow[t]{2}{*}{ Total síntomas positivos } & Mujer & 446 & 58.25 & 11.22 & \multirow{2}{*}{$-1.19(926,80)$} & \multirow[t]{2}{*}{.232} & \multirow[t]{2}{*}{-.077} \\
\hline & Varón & 483 & 59.17 & 12.33 & & & \\
\hline \multirow[t]{2}{*}{ Ind. Distrés Síntomas Positivos } & Mujer & 446 & 43.37 & 10.44 & \multirow{2}{*}{$-2.37(917,03)$} & .018 & \multirow[t]{2}{*}{-.550} \\
\hline & Varón & 481 & 45.15 & 12.36 & & & \\
\hline \multirow[t]{2}{*}{ Somatización } & Mujer & 397 & 51.38 & 10.99 & \multirow{2}{*}{$-2.51(752,36)$} & .012 & \multirow[t]{2}{*}{-.168} \\
\hline & Varón & 447 & 53.91 & 17.91 & & & \\
\hline \multirow[t]{2}{*}{ Obsesión-compulsión } & Mujer & 387 & 57.98 & 9.25 & \multirow{2}{*}{$-2.96(776,87)$} & .003 & \multirow[t]{2}{*}{-.208} \\
\hline & Varón & 409 & 60.15 & 11.36 & & & \\
\hline \multirow[t]{2}{*}{ Sensibilidad interpersonal } & Mujer & 402 & 57.72 & 11.02 & \multirow{2}{*}{$-0.93(796,86)$} & .353 & \multirow[t]{2}{*}{-.065} \\
\hline & Varón & 407 & 58.49 & 12.50 & & & \\
\hline \multirow[t]{2}{*}{ Depresión } & Mujer & 401 & 50.18 & 10.39 & \multirow{2}{*}{$-3.14(844,95)$} & .002 & \multirow[t]{2}{*}{-.214} \\
\hline & Varón & 448 & 52.62 & 12.19 & & & \\
\hline \multirow[t]{2}{*}{ Ansiedad } & Mujer & 402 & 53.62 & 10.53 & \multirow{2}{*}{$-2.38(847,67)$} & .018 & \multirow[t]{2}{*}{-.163} \\
\hline & Varón & 448 & 55.46 & 11.97 & & & \\
\hline \multirow[t]{2}{*}{ Hostilidad } & Mujer & 402 & 51.37 & 12.18 & \multirow{2}{*}{$-5.28(845)$} & .000 & \multirow[t]{2}{*}{-.363} \\
\hline & Varón & 445 & 55.88 & 12.58 & & & \\
\hline \multirow[t]{2}{*}{ Ansiedad Fóbica } & Mujer & 402 & 51.81 & 11.00 & \multirow{2}{*}{$0.21(825,67)$} & .832 & \multirow[t]{2}{*}{.014} \\
\hline & Varón & 442 & 51.63 & 13.96 & & & \\
\hline \multirow[t]{2}{*}{ Ideación Paranoide } & Mujer & 402 & 56.80 & 11.89 & & .011 & -.175 \\
\hline & Varón & 448 & 58.95 & 12.64 & $-2.55(848)$ & & \\
\hline Psicoticismo & Mujer & 400 & 57.83 & 11.73 & $-177(842)$ & .077 & -.121 \\
\hline & Varón & 444 & 59.33 & 12.77 & 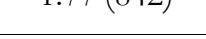 & & \\
\hline
\end{tabular}

bajas atendiendo a la clasificación de Cohen (1988). Las puntuaciones nos indican que esta muestra se encuentra en las agrupaciones de síntomas entre los percentiles 55 al 85, destacando las medias en ambos sexos en obsesión-compulsión y sensibilidad interpersonal. Las puntuaciones que figuran en la tabla corresponden a las puntuaciones T del SCL-90-R.

En cuanto a la edad, se halló que influía en todas las dimensiones psicopatológicas medidas: GSI $\left[X^{2}(120)=\right.$ $309.88, p<.001]$, PST [ $\left.X^{2}(126)=296.93, p<.001\right]$, PDSI $\left[X^{2}(126)=350.18, p<.001\right]$, Somatización $\left[X^{2}(108)=\right.$ 257.73, $p<.001]$, Obsesión-compulsión $\left[X^{2}(96)=206.59\right.$, $p<.001]$, Sensibilidad interpersonal $\left[X^{2}(78)=171,47, p<\right.$ $.001]$, Depresión $\left[X^{2}(120)=323.24, p<.001\right]$, Ansiedad $\left[X^{2}(90)=198.92, p<.001\right]$, Hostilidad $\left[X^{2}(84)=207.92\right.$, $p<.001]$, Ansiedad Total $\left[X^{2}(66)=156.68, \quad p<.001\right]$,
Ideación paranoide $\left[X^{2}(66)=241.13, p<.001\right]$ y Psicoticismo total $\left[X^{2}(60)=117.83, p<.001\right]$. Posteriormente, realizamos correlaciones entre edad y sintomatología, obteniendo correlaciones significativas y positivas en las variables: GSI $(r=.17, p<.001)$, PST $(r=.21, p<.001)$, Somatización $(r=.18, p<.001)$, Obsesión-compulsión $(r=.24, p<$ $.001)$, Sensibilidad interpersonal $(r=.21, p<.001)$, Depresión $(r=.21, p<.001)$, Ansiedad $(r=.19, p<.001)$, Hostilidad $(r=.17, p<.001)$, Ideación paranoide $(r=.17, p<$ $.001)$ y Escala adicional $(r=.10, p<.01)$ indicando que a mayor edad, mayor presencia de la sintomatología indicada, destacando la menor puntuación a los 12-13 años frente a los 17-18 años. No se hallaron diferencias estadísticamente significativas $(p<.05)$ en las dimensiones o síntomas psicopatológicos atendiendo al resto de variables sociodemográfi- 
cas ( $\mathrm{n}^{\mathrm{o}}$ de hermanos, situación laboral del padre y de la madre, tipo de hogar y estado civil de los padres)

Respecto de la variable Autoestima, no se hallaron diferencias significativas en base al sexo $[t(933)=-1.74, p=$ .082], aunque sí en base a la edad de los sujetos [ $X^{2}$ (156) $=362.32, p<.001]$, siendo la correlación débil y negativa $(r$ $=-.08$ ), alcanzando la puntuación más alta a los 12 años y la mínima a los 15 años. El resto de variables sociodemográficas no afectó a los resultados.

En cuanto al Estilo educativo de la madre, el sexo presentó diferencias en la dimensión de Afecto $[t(923)=4.70, p$ $<.001]$, Promoción $[t(923)=5.70, p<.001]$, Control conductual $[t(917)=5.58, p<.001]$, Control psicológico $[t$ $(921)=-2.56, p<.05]$, Revelación $[t(923)=9.29, p<.001]$ y Humor $[t(923)=5.14, \mathrm{p}<.001]$, alcanzando en todas ellas mayores puntuaciones las chicas que los chicos, excepto en control psicológico. La edad de los participantes afectó a las dimensiones del estilo educativo de la madre, en concreto con Afecto $\left[\left(X^{2}(186)=353.99, p<.001\right]\right.$, Promoción $\left[X^{2}\right.$ $(228)=440.14, p<.001]$, Control conductual $\left[X^{2}(174)=\right.$ $391.95, p<.001]$, Control psicológico $\left[X^{2}(234)=449,59, p\right.$ $<.001]$, Revelación $\left[X^{2}(150)=362.07, p<.001\right]$, Humor $\left[X^{2}(168)=379,45, p<.001\right]$. Las correlaciones fueron significativas y negativas en Afecto $(r=-.17, p<.001)$, Control conductual $(r=-.16, p<.001)$, Revelación $(r=-.15, p<$ $.001)$ y Humor $(r=-.15, p<.001)$, es decir, a menor edad, mejor percepción de esas dimensiones del estilo educativo de la madre, siendo la edad de los doce años la que mayores medias alcanzó frente al resto de las edades.

Respecto del Estilo educativo del padre, también se encontraron diferencias debidas al sexo en Promoción $[t$ (911) $=3.14, p<.01]$, Control conductual $[t(91)=4.64, p<.001]$, Control psicológico $[t(917)=-2.37, p<.05]$, Revelación $[t$ $(913)=5.61, p<.001]$ y Humor $[t(913)=3.21, p<.01]$, alcanzando una mejor percepción las chicas que los chicos. De igual modo, la edad también presentó diferencias en Afecto $\left[X^{2}(228)=475.01, p<.001\right]$, Promoción $\left[X^{2}(234)=\right.$ $492.81, p<.001]$, Control conductual $\left[X^{2}(180)=462.14, p\right.$ $<.001]$, Control psicológico $\left[X^{2}(228)=454.21, p<.001\right]$, Revelación $\left[X^{2}(150)=324.51, p<.001\right]$ y Humor $\left[X^{2}(180)\right.$ $=485,18, p<.001]$. Las correlaciones fueron: Afecto $(r=-$ $.19, p<.001)$, Control conductual $(r=-.18, p<.001)$, Revelación $(r=-.22, p<.001)$ y Humor $(r=-.19, p<.001)$, siendo mejor la percepción de los chicos/as de menor edad que los de mayor edad, destacando los 12 años frente al resto de las edades.

El tipo de hogar o con quien convivían los adolescentes presentó diferencias en algunas de las dimensiones del estilo educativo de la madre (Afecto, $p<.001$; Promoción, $p<$ .001 y Revelación, $p>$.001). En concreto, alcanzaron mayores puntuaciones cuando los adolescentes convivían con ambos padres o sólo con la madre frente a si vivían solos con el padre. No obstante estos datos debemos tomarlos con cautela por el bajo número de adolescentes que se encuentran en la condición de "solo padre". En el estilo educativo del padre, la variable Control conductual también pre- sentó diferencias debidas al tipo de hogar $[F(3,918=5.78 ; p$ $>$.01], siendo mayor el control percibido cuando el adolescente convivía con ambos padres que cuando lo hacía sólo con la madre.

\section{Correlaciones entre Síntomas psicopatológicos y Es- tilos parentales percibidos}

Para explorar la relación entre los Síntomas psicopatológicos de los adolescentes y los Estilos parentales percibidos llevamos a cabo correlaciones bivariadas (coeficiente de correlación de Pearson). La Tabla 3 presenta la matriz de correlaciones entre las mismas, separando el Estilo parental de la madre y el del padre. Podemos observar que la correlación entre Estilo materno y los distintos síntomas psicopatológicos y los índices globales es baja y media-baja, excepto con control psicológico que, como era de esperar, lo hace positivamente. El control conductual de la madre sólo presenta correlaciones significativas con los índices globales y con ansiedad y hostilidad. Centrándonos en las dimensiones psicopatológicas, destacamos las correlaciones entre Afecto y Obsesión-compulsión $(r=-.23, p<.001)$ y Afecto-Depresión $(r$ $=-.22, p<.001)$, Promoción y Obsesión-compulsión $(r=-$ $.18, p<.01)$, Control psicológico y Hostilidad $(r=.21, p<$ $.001)$, Revelación y Obsesión-compulsión $(r=-.25, p<$ $.001)$, Humor y Obsesión-compulsión $(r=-.25, \mathrm{p}<.001)$. En el caso del estilo del padre, las correlaciones entre las variables analizadas siguen la misma dirección que en el caso del estilo de las madres, es decir, todas correlacionan negativamente con los síntomas psicopatológicos excepto la variable Control psicológico que lo hace positivamente. El Control conductual de padre correlaciona con mayor número de variables que el de la madre. En concreto, está asociado a Obsesión-compulsión, Sensibilidad interpersonal, Depresión, Ansiedad, Hostilidad e Ideación paranoide. Las correlaciones más altas en cada dimensión son entre Afecto y Depresión $(r=-.29, p<.001)$, Promoción e Ideación paranoide $(r=-.20, p<.001)$, Control psicológico y Hostilidad $(r$ $=.19, p<.001)$, Revelación y Obsesión-compulsión $(r=$ $.29, p<.001)$ y Humor y Depresión $(r=-.26, p<.001)$.

\section{Correlaciones Síntomas psicopatológicos y Autoes- tima}

Las correlaciones entre Autoestima y síntomas psicopatológicos son significativas y negativas, indicando que a mayor Autoestima menor sintomatología. Destacan las correlaciones entre Autoestima-Depresión $(r=-.39, p>$.001) y Autoestima-Sensibilidad interpersonal $(r=-.32, p<.001)$.

Variables predictoras de los Síntomas psicopatológicos

Para examinar la capacidad predictiva de las variables Estilos parentales percibidos, Autoestima, sexo y edad sobre los Síntomas o respuestas psicopatológicas (factor) percibi- 
dos por los adolescentes se realizó un análisis de regresión lineal múltiple por pasos. Como podemos observar en la Tabla 4 los porcentajes de varianza explicada (coeficientes de determinación ajustados) por la variables predictoras fueron, en la mayor parte, de magnitud baja y media-baja (5.6\% a
26.7\%). Las dimensiones psicopatológicas más explicadas por las variables predictoras han sido Depresión (26.7\%), Índice global de gravedad (21.1\%), Sensibilidad interpersonal (19.7\%) y Obsesión-compulsión (19.0\%).

Tabla 3. Matriz de Coeficientes de correlación de Pearson entre Estilos parentales percibidos y sintomatología psicopatológica. Estilo materno percibido

\begin{tabular}{|c|c|c|c|c|c|c|}
\hline & Afecto & Promoción & Control conductual & Control psicológico & Revelación & Humor \\
\hline Índice Global Gravedad & $-.22 * *$ & $-.17 * *$ & $-.09 * *$ & $.19 * *$ & $-.22 * *$ & $-.23 * *$ \\
\hline Total síntomas positivos & $-.23 * *$ & $-.13^{* *}$ & $.09 * *$ & $.16^{* *}$ & $-.19 * *$ & $-.23 * *$ \\
\hline Ind. Distrés Síntomas Positivos & $-.10^{* *}$ & $-.11 * *$ & $-.07 *$ & $.12^{* *}$ & $-.10 * *$ & n.s \\
\hline Somatización & $-.12 * *$ & $-.13 * *$ & n.s & $.09 * *$ & $-.13 * *$ & $-.15^{* *}$ \\
\hline Obsesión-compulsión & $-.23 * *$ & $-.18^{* *}$ & n.s & $.21 * *$ & $-.25^{* *}$ & $-.25^{* *}$ \\
\hline S. interpersonal & $-.21 * *$ & $-.14 * *$ & n.s & $.18^{* *}$ & $-.21 * *$ & $-.21 * *$ \\
\hline Depresión & $-.22 * *$ & $-.16^{* *}$ & n.s & $.20 * *$ & $-.22 * *$ & $-.20 * *$ \\
\hline Ansiedad & $-.19 * *$ & $-.12 * *$ & $-.11 * *$ & $.16^{* *}$ & $-.16^{* *}$ & $-.19 * *$ \\
\hline Hostilidad & $-.21 * *$ & $-.12 * *$ & $-.11 * *$ & $.21 * *$ & $-.22 * *$ & $-.19 * *$ \\
\hline Ansiedad fóbica & $-.10 * *$ & $-.10 * *$ & n.s & $.11^{*}$ & $-.07^{*}$ & $-.12 * *$ \\
\hline Ideación paranoide & $-.21 * *$ & $-.16^{* *}$ & n.s & $.19^{* *}$ & $-.22 * *$ & $-.21 * *$ \\
\hline Psicoticismo & $-.14 * *$ & $-.12 * *$ & n.s & $.18^{* *}$ & $-.12 * *$ & $-.15^{* *}$ \\
\hline
\end{tabular}

Estilo paterno percibido

\begin{tabular}{|c|c|c|c|c|c|c|}
\hline & Afecto & Promoción & Control conductual & Control psicológico & Revelación & Humor \\
\hline Índice Global Gravedad & $-.29 * *$ & $-.19 * *$ & $-.19 * *$ & $.15^{* *}$ & $-.29 * *$ & $-.25^{* *}$ \\
\hline Total síntomas positivos & $-.25^{* *}$ & $-.13^{* *}$ & $-.14 * *$ & $.13^{* *}$ & $-.27 * *$ & $-.20 * *$ \\
\hline Ind. Distrés Síntomas Positivos & $-.16^{* *}$ & $-.13^{* *}$ & n.s & $.09 * *$ & $-.09 * *$ & $-.08^{*}$ \\
\hline Somatización & $-.14 * *$ & $-.14 * *$ & n.s & $.09 *$ & $-.17 * *$ & $-.15^{* *}$ \\
\hline Obsesión-compulsión & $-.25^{* *}$ & $-.16^{* *}$ & $-.12^{* *}$ & $.14^{* *}$ & $-.29 * *$ & $-.20 * *$ \\
\hline Sensibilidad interpersonal & $-.28 * *$ & $-.18 * *$ & $-.16^{* *}$ & $.13^{* *}$ & $-.26 * *$ & $-.24 * *$ \\
\hline Depresión & $-.29 * *$ & $-.19 * *$ & $-.17 * *$ & $.15^{* *}$ & $-.28 * *$ & $-.26 * *$ \\
\hline Ansiedad total & $-.24 * *$ & $-.14 * *$ & $-.17 * *$ & $.13^{* *}$ & $-.21 * *$ & $-.20 * *$ \\
\hline Hostilidad & $-.27 * *$ & $-.18 * *$ & $-.19 * *$ & $.19^{* *}$ & $-.31 * *$ & $-.19 * *$ \\
\hline Ansiedad fóbica & $-.09 * *$ & n.s & n.s & $.08^{*}$ & n.s & n.s \\
\hline Ideación paranoide & $-.27 * *$ & $-.20 * *$ & $-.14 * *$ & $.15^{* *}$ & $-.29 * *$ & $-.19 * *$ \\
\hline Psicoticismo & $-.19 * *$ & $-.11 * *$ & n.s & $.12^{* *}$ & $-.18 * *$ & $-.17 * *$ \\
\hline
\end{tabular}

$* * p<.01 ; * p<.05$

La variable Autoestima resultó predictora en sentido negativo tanto en los índices globales como en el resto de síntomas psicopatológicos, obteniendo el mayor peso en dos de los índices generales (Índice global de Severidad, Beta = .265; Total Síntomas Positivos, Beta $=-.241)$ y en seis dimensiones sintomáticas (Somatización, Beta $=-.222$; Sensibilidad Interpersonal, Beta $=-.267$; Depresión, Beta $=-.348$; Ansiedad; Beta $=$-.203; Ansiedad Fóbica, Beta $=$-.219; Psicoticismo, Beta $=-.148$ ). La edad y el sexo son variable predictoras de los índices de salud, tanto en los índices generales y en 7 de las 9 agrupaciones sintomáticas (en el caso de la edad) y en 5 (en el caso del sexo).

Respecto de las dimensiones de los Estilos parentales percibidos, la Revelación padre está presente como predictor en el Índice Global de Gravedad (Beta = -.117), Total de síntomas positivos (Beta $=-.196)$, Obsesión-compulsión (Beta $=$ -.196), Sensibilidad interpersonal (Beta $=-.112)$, Depresión
$($ Beta $=-.091)$, Hostilidad $($ Beta $=-.137)$, Ideación paranoide $($ Beta $=-.174)$ y Psicoticismo (Beta $=-.098)$, siendo en dos de ellos el de mayor peso, tal y como podemos observar en la tabla 5. El signo negativo indica la relación inversa, es decir, a mayor revelación, menor sintomatología. El Control psicológico madre también está presente como predictor en varias de las variables de salud, en concreto en el Índice Global de Gravedad (Beta $=.124)$, Total de síntomas positivos (Beta $=.132)$, Obsesión-compulsión $($ Beta $=.172)$, Sensibilidad interpersonal $($ Beta $=.120)$, Depresión $($ Beta $=.122)$, Ansiedad $($ Beta $=.118)$, Hostilidad (Beta $=.146)$, Ideación paranoide $($ Beta $=.131)$ y Psicoticismo $($ Beta $=.128)$. El signo positivo indica que a mayor control psicológico mayor sintomatología. Otras dimensiones de los estilos parentales, tales como Afecto padre, Control conductual padre y madre y Promoción madre son predictores en algunos de los síntomas pero en menor medida que los anteriores. 
Tabla 4. Resultados del análisis de regresión por pasos en la sintomatología psicopatológica.

\begin{tabular}{|c|c|c|c|c|}
\hline & & Beta & $t$ & $\mathrm{R}^{2}$ corregida \\
\hline \multirow[t]{6}{*}{ Índice Global Gravedad } & Autoestima & -.265 & $-8.667 * * *$ & .211 \\
\hline & Control psicológico madre & .124 & $4.070^{* * *}$ & \\
\hline & Sexo & .125 & $4.094 * * *$ & \\
\hline & Afecto padre & -.136 & $-3.520 * * *$ & \\
\hline & Revelación padre & -.117 & $-3.028 * *$ & \\
\hline & Edad & .097 & $3.148^{* *}$ & \\
\hline \multirow[t]{4}{*}{ Total síntomas positivos } & Autoestima & -.241 & $-7.828 * * *$ & .177 \\
\hline & Revelación padre & -.196 & $-6.174 * * *$ & \\
\hline & Edad & .155 & $4.980^{* * *}$ & \\
\hline & Control psicológico madre & .132 & $4.292^{* * *}$ & \\
\hline \multirow[t]{5}{*}{ Ind. Distrés Síntomas Positivos } & Edad & -.223 & $-6.868^{* * *}$ & .102 \\
\hline & Afecto padre & -.173 & $-5.234 * * *$ & \\
\hline & Autoestima & -.151 & $-4.643^{* * *}$ & \\
\hline & Sexo & .116 & $3.602 * * *$ & \\
\hline & Control conductual madre & .074 & $2.254 *$ & \\
\hline \multirow[t]{4}{*}{ Somatización } & Autoestima & -.222 & $-6.568 * * *$ & .096 \\
\hline & Edad & .158 & $4.706^{* * *}$ & \\
\hline & Promoción madre & -.103 & $-3.001 * *$ & \\
\hline & Sexo & .082 & $2.377^{*}$ & \\
\hline \multirow[t]{5}{*}{ Obsesión-Compulsión } & Revelación padre & -.196 & $-5.635^{* * *}$ & .190 \\
\hline & Autoestima & -.195 & $-5.872 * * *$ & \\
\hline & Edad & .186 & $5.533^{* * *}$ & \\
\hline & Control psicológico madre & .172 & $5.212^{* * *}$ & \\
\hline & Sexo & .079 & $2.356^{*}$ & \\
\hline \multirow[t]{5}{*}{ Sensibilidad Interpersonal } & Autoestima & -.267 & $-8.172^{* * *}$ & .197 \\
\hline & Edad & .162 & $4.878^{* * *}$ & \\
\hline & Control psicológico madre & .120 & $3.627 * * *$ & \\
\hline & Afecto padre & -.113 & $-2.763 * *$ & \\
\hline & Revelación padre & -.112 & $-2.766^{* *}$ & \\
\hline \multirow[t]{6}{*}{ Depresión } & Autoestima & -.348 & $-11.293 * * *$ & .267 \\
\hline & Edad & .151 & $4.862 * * *$ & \\
\hline & Control psicológico madre & .122 & $3.981 * * *$ & \\
\hline & Afecto padre & -.131 & $-3.341 * *$ & \\
\hline & Sexo & .110 & $3.532 * * *$ & \\
\hline & Revelación padre & -.091 & $-2.303^{*}$ & \\
\hline \multirow[t]{6}{*}{ Ansiedad } & Autoestima & -.203 & $-6.100 * * *$ & .139 \\
\hline & Edad & .131 & $3.918^{* * *}$ & \\
\hline & Afecto padre & -.124 & $-3.521 * *$ & \\
\hline & Control psicológico madre & .118 & $3.343 * * *$ & \\
\hline & Control conductual padre & -.096 & $-2.676^{* *}$ & \\
\hline & Sexo & .073 & $2.205^{*}$ & \\
\hline \multirow[t]{6}{*}{ Hostilidad } & Control psicológico madre & .146 & $4.424 * * *$ & .157 \\
\hline & Revelación padre & -.137 & $-.3 .224 * *$ & \\
\hline & Sexo & .136 & $4.089 * * *$ & \\
\hline & Edad & .114 & $3.418^{* * *}$ & \\
\hline & Autoestima & -.103 & $-3.113 * *$ & \\
\hline & Afecto padre & -.121 & $-2.895^{* *}$ & \\
\hline \multirow[t]{2}{*}{ Ansiedad fóbica } & Autoestima & $-.219 * * *$ & -6.398 & .056 \\
\hline & Promoción madre & $-.088^{* *}$ & -2.568 & \\
\hline \multirow[t]{5}{*}{ Ideación paranoide } & Revelación padre & -.174 & $-4.171 * * *$ & .144 \\
\hline & Autoestima & -.148 & $-4.493 * * *$ & \\
\hline & Control psicológico madre & .131 & $3.983 * * *$ & \\
\hline & Edad & .110 & $3.286^{* *}$ & \\
\hline & Afecto padre & -.089 & $-2.122 *$ & \\
\hline \multirow[t]{4}{*}{ Psicoticismo } & Autoestima & -.148 & $-4.330 * * *$ & .084 \\
\hline & Control psicológico madre & .128 & $3.741 * * *$ & \\
\hline & Revelación padre & -.098 & $-2.207^{*}$ & \\
\hline & Afecto padre & -.096 & $-2.207^{*}$ & \\
\hline
\end{tabular}




\section{Discusión y conclusiones}

El objetivo general de nuestro estudio ha sido analizar la interacción de los Estilos parentales percibidos (en sus diferentes dimensiones), la Autoestima y los Síntomas o repuestas psicopatológicas mostradas en una muestra comunitaria de adolescentes españols. Para alcanzar este objetivo se seleccionó una amplia muestra de adolescentes $(N=935)$ de 12 a 18 años que fueron evaluados en las variables Autoestima, Estilos parentales percibidos y Síntomas psicopatológicas.

Las medias obtenidas (en puntuaciones T) en los síntomas psicopatológicos evaluadas con el SCL-90-R en la muestra indicaron que los adolescentes se hallaban entre los percentiles 55 al 85, atendiendo al baremo aportado por el test en población no clínica (González et al., 2002), destacando las alcanzadas en Obsesión-compulsión y Sensibilidad interpersonal en ambos sexos, coincidiendo en parte con los resultados de Kim (2003).

Centrándonos en el primer objetivo propuesto, se analizó la existencia de diferencias en Síntomas psicopatológicos, Autoestima y Estilos parentales percibidos debidas a las variables sexo, edad y tipo de hogar. En concreto, y respeto de la variable edad, encontramos que los adolescentes con mayor edad (17-18 años) presentaban mayores puntuaciones en síntomas psicopatológicos frente a los de 12-13 años, coincidiendo en parte con los resultados obtenidos por Garaigordobil et al. (2008), indicando que la adolescencia tardía es un período crítico donde se plantean problemas y toma de decisiones importantes que pueden afectar en su salud mental. De igual modo, la autoestima de los chicos/as de 12 años alcanzó una media mayor que la obtenida por el grupo de 15 años. Esto puede ser debido a que los 15 años el adolescente sufre una fuerte crisis de identidad, volviéndose más crítico, irascible e intolerante con él mismo y con su entorno. Los estilos educativos percibidos presentaron también diferencias atendiendo a la edad, de modo que a los 12 años el adolescente percibe mejor a los padres que desde los 13 a 18 años, siendo más críticos con éstos en esta última etapa.

En la mayor parte de las dimensiones psicopatológicas, excepto en Sensibilidad interpersonal, Ansiedad fóbica y Psicoticismo, alcanzaron mayores puntuaciones medias (aunque con un bajo tamaño del efecto) los varones frente a las mujeres, no coincidiendo en este caso con el estudio de Garaigordobil et al. (2008), quizás debido a que en la investigación de estos autores los análisis fueron realizados con puntuaciones directas, no transformadas a puntuaciones $T$, lo cual explicaría las diferencias encontradas. Atendiendo a la variable autoestima, nuestros resultados coinciden con los de los autores mencionados anteriormente y otros (Bragado et al., 2008) ya que no se hallaron diferencias en base al sexo. En cuanto a los estilos educativos, observamos que las chicas percibieron mejor a las madres y a los padres en todas las dimensiones positivas, excepto en la dimensión considerada negativa de control psicológico, coincidiendo con los resultados hallados por Oliva et al. (2007).

Otra variable que presentó diferencias en los resultados fue el tipo de familia/hogar, de modo que cuando los adolescentes vivían con ambos padres o sólo con la madre, percibían a la madre más afectuosa, más impulsora de la autonomía y, por tanto, se sentían con más deseos de compartir sus vivencias, que cuando convivían sólo con el padre. De igual modo, cuando convivían con ambos padres, percibían a los padres más controladores conductualmente que cuando vivían sólo con la madre (resultado lógico ya que se trata del control del padre)

El segundo de nuestros objetivos fue evaluar la relación entre los Estilos parentales percibidos, la Autoestima y los Síntomas psicopatológicos, afirmando que correlacionarían significativamente. Analizando por separado el Estilo educativo de la madre y el del padre, pudimos observar que las dimensiones del estilo educativo de la madre (excepto control conductual) y los síntomas e índices globales de salud correlacionaron negativa y significativamente. Control psicológico y dimensiones psicopatológicas presentaron correlaciones positivas (a mayor control, mas síntomas). En el caso del estilo educativo del padre, las correlaciones entre las distintas dimensiones y los síntomas de salud fueron negativas excepto la variable control psicológico que lo hizo positivamente. Respecto del control conductual del padre y la salud de los chico/as, fue mayor el número de variables relacionadas significativamente que en el caso de la percepción materna. Por tanto, nuestra segunda hipótesis quedó confirmada, es decir a mayor afecto, promoción, control conductual, revelación y humor menor puntuación en síntomas psicopatológicos, mientras que a mayor control psicológico, mayor número de síntomas, coincidiendo nuestros resultados con los de investigaciones anteriores (Gracia et al., 2005; Hasebe et al., 2004; Kerr y Stattin, 2000; Oliva et al., 2007).

La autoestima y las dimensiones psicopatológicas correlacionaron negativa y significativamente, siguiendo la dirección de lo apuntado por autores anteriores (Dowd, 2002; Garaigordobil et al., 2005; Garaigordobil et al., 2008; González y Rodríguez, 1993; Kim, 2003).

El último objetivo de nuestro estudio fue examinar la capacidad predictiva de las variables mencionadas en la salud psicopatológica de los adolescentes, destacando la relevancia de la autoestima, control psicológico y revelación percibida. Como dato importante a tener en cuenta es que los porcentajes de varianza explicada por la variables predictoras fueron, en la mayor parte, de magnitud baja y media-baja (5.6\% a $26.7 \%$ ), lo cual nos lleva a plantearnos la necesidad de tener en cuenta otro tipo de variables, tal y como recomienda la Psicopatología del Desarrollo (Cichetti y Rogosch, 2002), analizando factores personales y contextuales que pueden influir en el inicio o mantenimiento del problema.

Los predictores más importantes fueron: Autoestima, Edad y Sexo, Revelación y Afecto del padre, y Control psicológico de la madre. La variable Autoestima resultó ser un 
buen predictor presente tanto en los índices globales como en el resto de síntomas psicopatológicos, obteniendo el mayor peso negativo en la mayoría de los síntomas, destacando en Depresión y Sensibilidad Interpersonal. Nuestros datos coinciden con otros estudios anteriores sobre la relación de este constructo con la salud de los adolescentes (Garaigordobil et al., 2005; 2008; Kim, 2003; Taylor et al., 2012, etc.).

El control psicológico de la madre es un buen predictor de problemas psicológicos según nuestros datos, que apoyan los obtenidos por otros autores (Barber et al., 1994; Oliva et al., 2008). Por tanto, los adolescentes que perciben a sus madres como chantajistas y manipuladoras podrían presentar peores índices de salud y ajuste mental. Por el contrario, la revelación o manifestación del adolescente de sus inquietudes hacia el padre estaría relacionado con menores síntomas psicopatológicos, en la dirección de Kerr y Stattin (2000) que indicaron que la revelación se relacionaba con un buen ajuste, mientras que los esfuerzos de control y la vigilancia de los padres estaban vinculados a una deficiente adaptación. De igual modo, coinciden con los resultados presentados por Hasebe et al. (2004) ya que cuando los adolescentes comunican su mundo más privado, de forma espontánea pueden presentar índices de mayor salud mental. Pese a que la variable Afecto padre y madre no hayan aparecido como predictores, sí están presentes debido a que Revelación, Afecto y Promoción del padre presentaban una alta correlación entre sí, al igual que Revelación, Afecto y Promoción de la madre. Por otro lado, el Control psicológico de la madre estaba altamente relacionado con el Control psicológico del padre. De acuerdo con Oliva et al. (2008), podemos resumir que los estilos educativos percibidos de ambos padres están relacionados con los síntomas psicopatológicos de los adolescentes.

Por tanto, las variables Autoestima, Estilos educativos percibidos (control psicológico de la madre y revelación y afecto del padre), Sexo y Edad son predictoras de la síntomas psicopatológicos de los adolescentes de 12 a 18 años. No obstante, esta conclusión debe ser matizada teniendo en

\section{Referencias}

Baumrind, D. (1968). Authoritarian vs. authoritative parental control. Adolescence, 3, 255-272.

Baumrind, D. (1971). Current patterns of parental authority. Developmental Psychology Monograph, 4, 1-102. http://dx.doi.org/10.1037/h0030372

Barber, B. K., Olsen, J. E. y Shagle, S. C. (1994). Associations between parental psychological and behavioral control and youth internalized and externalized behaviors. Child Development, 65, 1120 - 1136. http://dx.doi.org/10.2307/1131309

Bragado, C., Hernández, M. J., Sánchez, M. L. y Urbano, S. (2008). Autoconcepto físico, ansiedad, depresión y autoestima en niños con cáncer y niños sanos sin historia de cáncer. Psicothema, 20, 413-419.

Boix, M., Rosa-Alcázar, A. I. y Olivares-Olivares, P.J. (2011). Papel de la focalización de la atención en el tratamiento de la fobia social generalizada en adolescentes. Anales de Psicología, 27, 718-728.

Cichetti, D. y Rogosch, F. A. (2002). A developmental psychopathology perspective on adolescence. Journal of Consulting and Clinical Psychology, 70, 610. http://dx.doi.org/10.1037//0022-006X.70.1.6 cuenta que se trata de un estudio de carácter transversal y correlacional lo que nos impide hablar de relación causal. No obstante, estos resultados son interesantes de cara a la prevención de problemas de salud y ajuste psicológico del adolescente, ya que las relaciones familiares conflictivas, al igual que la baja autoestima pueden ser factores de riesgo, que trabajados adecuadamente, pueden transformarse en factores de protección importantes en el mantenimiento o disminución de un patrón de comportamiento disfuncional (Lemos, 2003). Además, y coincidiendo con Oliva (2006), los padres deben saber el momento evolutivo de sus hijos y la importancia de los estilos parentales adecuados, creando un ambiente que invite a la revelación y elimine el control psicológico.

Una de las limitaciones del estudio es que el procedimiento de selección de la muestra inicial no fue aleatorio, sino de centros voluntarios que se incluyeron posteriormente al azar atendiendo al tipo de titularidad (pública vs. concertada). Una mejora del estudio que nos permitiría aumentar la validez de los resultados sería llevarlo a cabo en más Comunidades Autónomas e invitar a los centros seleccionados previamente al azar. Otra limitación ha sido que sólo hemos contado con los autoinformes de los adolescentes, pudiendo haber sido importante utilizar el informe de terceros (padres, profesores y amigos). No obstante, la información aportada de los adolescentes es la que ellos viven, independientemente de que sea más o menos objetiva.

Las limitaciones mencionadas pueden transformase en perspectivas de trabajo futuras junto a otras investigaciones que permitan analizar elementos personales y contextuales para entender más y mejor el inicio y mantenimiento de los problemas de salud mental de los niños/adolescentes. Estos estudios podrían llevarse a cabo en población sana o enferma, ser estudios transversales o longitudinales.

Agradecimientos.- Al Profesor A. Oliva (Universidad Sevilla) por el envío de materiales e instrumentos que hemos solicitado.

Cohen, J. (1988). Statistical power analysis for the behavioral sciences. Hillsdale, N.J.: Erlbaum.

Cuervo, A. (2010). Pautas de crianza y desarrollo socioafectivo en la infancia. Diversitas: Perspectivas en Psicologia, 6, 111-121.

Derogatis, L. R. (2002). SCL-90-R. Cuestionario de 90 sintomas revisado. Madrid: TEA (original work published in 1983).

Dowd, S. A. (2002). Internalizing symptoms in adolescents: Assessment and relationship to self-concept. Dissertation Abstract International Section B: The Sciences and Engineering, 62 (8-B), 3796.

Estévez, A. y Calvete, E. (2007). Esquemas cognitivos en personas con conducta de juego patológico y su relación con experiencias de crianza. Clinica y Salud, 18, 23-43.

Estévez, E., Musitu, G. y Herrero, J. (2005). El rol de la comunicación familiar y del ajuste escolar en la salud mental del adolescente. Salud Mental, 28, 81-89.

Fieldt, T., Diego, M. y Sanders, C. (2001) Adolescent depression and risk factors. Adolescence, 43, 491-498. 
Florenzano, R., Valdés, M., Cáceres, E., Santander, S., Aspillaga, C. y Musalem, C. (2011). Relación entre ideación suicida y estilos parentales en un grupo de adolescentes chilenos. Revista Médica Chile, 139, 1529-1533. http://dx.doi.org/10.4067/S0034-98872011001200001

Garaigordobil, M., Durá, A., y Pérez, J.I. (2005). Psychopathological symptoms, behavioural problems, and selfconcept/self-esteem: A study with adolescents from 14 to 17 years. Annuary of Clinical and Health Psychology, 1, 53-63.

Garaigordobil, M., Pérez, J. I. y Mozas, M. (2008). Self-concept, self-esteem and psychopathological symptoms. Psicothema, 20, 114-123.

González, C. y Rodríguez, E. (1993). Autoestima y sintomatología depresiva en adolescentes mexicanos: Un estudio correlacional por género. Revista ABP-APAL, 15, 59-63.

González, J. L., De las Cuevas, C., Rodríguez, M. y Rodríguez, F. (2002). SCL-90-R, Symptom Cheklist 90 Revised, Spanish adaptation. Madrid: TEA.

Gracia, E., Lila, M. y Musitu, G. (2005). Rechazo parental y ajuste psicológico y social de los hijos. Salud Mental, 28, 73-81.

Hasebe, Y., Nucci, L. y Nucci, M. (2004). Parental control of the personal domain and adolescent symptoms of psychopathology: A crossnational study in the United States and Japan. Child Development, 75, 114. http://dx.doi.org/10.1111/j.1467-8624.2004.00708.x

Hedges, L. V. y Olkin, I. O. (1985). Statistical Methods for Meta-Analysis. Orlando, FL: Academic Press.

Heider, D., Matschinger, H., Bernet, S., Alonso, J. y Angermeyer, M. (2006). Relationship between parental bonding and mood disorder in six European countries. Psychiatry Research, 143, 89-98.

Iglesias, B. y Romero, E. (2009). Estilos parentales percibidos, psicopatología y personalidad en la adolescencia. Revista de Psicopatología y Psicología Clínica, 14, 63-77.

Jiménez, T, Musitu, G. y Murgui, S. (2008). Funcionamiento familiar y consumo de sustancias en adolescentes: El rol mediador de la autoestima. International Journal of Clinical and Health Psychology, 8, 139-151. http://dx.doi.org/10.1174/021347406775322214

Kerr, M. y Stattin, H. (2000). What Parents know, How They Know it, and Several Forms of Adolescent Adjustment: Further Support for a Reinterpretation of Monitoring. Developmental Psychology, 36, 366-380.

Kim, Y. H. (2003). Correlation of mental health problems with psychological constructs in adolescence: Final results from a 2-year study. International Journal of Nursing Studies, 40, 115-124. http://dx.doi.org/10.1016/S0020-7489(02)00037-8

Krueger, R. F. y Piasecki, T.M. (2002). Toward a dimensional and psychometrically-informed approach to conceptualizing psychopathology. Behaviour Research and Therapy, 40, 485-499. http://dx.doi.org/10.1016/S0005-7967(02)00016-5

Lemos, S. (2003). La psicopatología de la infancia y la adolescencia: Consideraciones básicas para su estudio. Papeles del psicólogo, 85, 19-28.

Liu, Y. (2003). Parent-child interaction and children's depression: the relationship between parent-child interaction and children's depressive symptoms in Taiwan. Journal Adolescence, 26, 447-457. http://dx.doi.org/10.1016/S0140-1971(03)00029-0

Maccoby, E. E. y Martin, J. A. (1983). Socialization in the context of the family: parent child interaction. En P. H. Mussen y E. M. Hetherington (Eds.), Handbook of child psychology: Vol. 4. Socialization, personality and social development (pp. 1-101). New York, Wiley (4a. edición).

Martín-Albo, J., Nuñez, J. L., Navarro, J. y Grijalvo (2007). The Rosenberg Self-Esteem Scale: Translation and Validation in University Students. The Spanish Journal of Psychology, 10, 458-467.

Moreno, M., González y Ortiz, G. (2009). Trastorno Alimentario y su relación con la Imagen Corporal y la Autoestima en Adolescentes. Terapia Psicológica, 27, 181-190.

Musitu, G., Jiménez, T. I. y Murgui, S. (2007). Funcionamiento familiar, autoestima y consumo de sustancias en adolescentes: un modelo de mediación. Salud pública de México, 49, 3-10. http://dx.doi.org/10.1590/S0036-36342007000100002

Muris, P., Meesters, C., Morren, M. y Moorman, L. (2004). Anger and hostility in adolescents: relationship with self-reported attachment style and perceived parental rearing styles. Journal of Psychosomatic Research, 57, $257-$ 64. http://dx.doi.org/10.1016/S0022-3999(03)00616-0

Oliva, A. (2006). Relaciones familiares y desarrollo adolescente. Anuario de Psicología, 37, 209-223.

Oliva, A., Parra, A. y Arranz, E. (2008). Estilos relacionales parentales y ajuste del adolescente. Infancia y Aprendizaje, 31, 93-106. http://dx.doi.org/10.1174/021037008783487093

Oliva, A., Parra, A., Sánchez, I. y López, F. (2007). Estilos educativos materno y paterno: evaluación y relación con el ajuste del adolescente. Anales de Psicologia, 25, 49-56.

Parra, A. y Oliva, A. (2006). Un análisis dimensional sobre las dimensiones relevantes del estilo parental durante la adolescencia. Infancia y Aprendizaje, 29, 453-470.

Pérez, P. y Canovas, C. (1994). Valores y estilos familiares de educación. En Fundación Santa María (Ed.), V alores y pautas de crianza familiar. El niño de 0 a 6 años. Estudio interdisciplinary (pp. 113-158). Madrid: S.M.

Ramírez, M. (2002). Prácticas de crianza de riesgo y problemas de conducta en los hijos. Apuntes de Psicología, 20, 273-282.

Richard de Minzi, M. (2005). Estilos parentales y estrategias de afrontamiento en niños. Revista Latinoamericana de Psicología, 37, 47-58.

Rosenberg, M. (1965). Society and the adolescent self-image. Princeton, NJ: Princeton University Press.

Sáez, E. y Rosselló, J. (2001) Relación entre el ambiente familiar, los síntomas depresivos y los problemas de conducta en adolescentes puertorriqueños/as. Revista Interamericana de Psicología, 35, 113-125.

Schwab-Stone, M. E. y Briggs-Growan, M. J. (1998). The scope and prevalence of psychiatric in childhood and adolescence. En P. Ferrari (Ed.), Designing mental health services and systems for children and adolescents: A shrewd investment (pp. 2-25). Philadelphia: Brunner/Mazel.

Taylor, A., Wilson, C., Slater, A. y Mohr, P. (2012) Self-esteem and body dissatisfaction in young children: Associations with weight and perceived parenting style. Clinical Psychologist, 16, 25-35. http://dx.doi.org/10.1111/j.1742-9552.2011.00038.x

(Articulo recibido: 29-12-2012; revisado: 10-05-2013; aceptado: 09-06-2013) 